\title{
A Case of Post-partum Chronic Subdural Hematoma.
}

\author{
Suyasha Rajbhandari ${ }^{1}$, Pritam Gurung ${ }^{1}$, Gopi Nepal ${ }^{1}$, Samir Acharya ${ }^{1}$, and Basant Pant ${ }^{1}$ \\ ${ }^{1}$ Annapurna Neurological Institute and allied Sciences
}

June 24, 2021

\begin{abstract}
Intracranial subdural hematoma following spinal anesthesia is an infrequent occurrence and has variable presentation. Due to rarity, it may often be misdiagnosed as post-dural puncture headache. In this report, we describe a case of a 25-year-old woman who presented with a headache following lower segment cesarean section after spinal anesthesia.
\end{abstract}

\section{A Case of Post-partum Chronic Subdural Hematoma.}

\section{Authors:}

Suyasha Rajbhandari M.B.B.S ${ }^{1}$

Pritam Gurung, M.D, Ph.D ${ }^{1}$

Gopi Nepal, M.B.B.S ${ }^{1}$

Samir Acharya, M.S ${ }^{1}$

Basant Pant, M.D.,Ph.D ${ }^{1}$

1 = Department of Neurosurgery, Annapurna Neurological Institute and allied Sciences, Maitighar, Kathmandu, Nepal.

\section{Corresponding Author:}

Suyasha Rajbhandari

Annapurna Neurological Institute and allied Sciences, 44700, Kathmandu, Nepal

Email: suyasharajbhandari1882@gmail.com

Phone No.: +977-9849620285

\section{INTRODUCTION:}

Spinal anesthesia is commonly used anesthesia during obstetric procedures. Although complications related to spinal anesthesia are generally benign, about $0.05 \%$ of complications are critical ${ }^{1,2}$. Post-dural puncture headache $(\mathrm{PDPH})$ and hypotension are the most common complication of spinal anesthesia ${ }^{3}$.

Intracranial subdural hematoma (ISH) is one of the rare but fatal complications of spinal anesthesia ${ }^{1}$. ISH following spinal anesthesia is reported to be more common in the obstetric population. The incidence rate of ISH following neuraxial anesthesia in the obstetric population is $0.0002 \%^{2}$.

The most common presentation of intracranial subdural hematoma is headache (74-91\%) ${ }^{2,4}$. When headache is the presenting complaint in the puerperal period, ISH is rarely presumed, leading to misdiagnosis and delay in treatment. Although an infrequent complication, early diagnosis of this disease manifested by headache is 
crucial during the post-partum period to avoid potentially fatal complications that will impact both mother and the baby.

This study aims to report a rare case of chronic subdural hematoma (CSDH) following lower segment cesarean section (LSCS) delivery after spinal anesthesia and discuss the measures we can take for early diagnosis and prevention of potentially fatal complications.

\section{CASE PRESENTATION:}

A 25-year-old woman presented to our emergency department with a complaint of persistent progressive, non-postural headache for 3 days. The headache was insidious in onset and was not associated with nausea, vomiting, impairment of consciousness, focal weakness, or numbness. Sixteen days prior to the presentation, she had delivered a baby via LSCS after spinal anesthesia. She had no history of pre-eclampsia, head trauma, connective tissue disorder during her pregnancy, and preoperative hematological, serological, and coagulation studies were normal. Following the spinal anesthesia and LSCS, she did not have any reported complications. Her neurological examination at the time of presentation was unremarkable.

Computed tomography (CT) scan of the head was performed which revealed up to $0.9 \mathrm{~cm}$ thick hypodense $\mathrm{CSDH}$ in right fronto-parieto-temporal convexity with some degree of mass effect and left-sided midline shift measuring about $0.6 \mathrm{~cm}$ from the center (Figure 1). The cerebral CT arteriogram and cerebral venogram study were normal. Preoperative hematological and coagulation studies were normal. The patient subsequently underwent a right frontoparietal burr hole and evacuation of the hematoma under general anesthesia without complications. On her third postoperative day, the patient was discharged home.

\section{DISCUSSION:}

ISH is a rare complication of spinal anesthesia. About $41 \%$ of postpartum ISH becomes chronic and the ratio of acute $(31 \%)$ and subacute $(33 \%)$ subdural hematomas (SDH) are similar ${ }^{3}$.

While the exact incidence of ISH following spinal anesthesia is unknown, as patients are usually treated conservatively without any investigations and reporting, it is known that the incidence of intracranial subdural hematoma following this anesthesia is 1:500,000-1,000,000 ${ }^{5}$.

The mechanism of development of ISH following spinal anesthesia is analogous to PDPH. It is postulated that the orifice made in the dura mater after puncturing with the cerebrospinal fluid (CSF) needle, remains open for several weeks after the puncture. This leads to rapid loss of CSF which reduces the intraspinal and intracranial pressure. This causes a caudal shift of the brain, which causes traction of the structures sensitive to pain and bridging veins in the subdural space leading to its rupture causing ISH and headache ${ }_{5,6}$. The time taken for diagnosis of ISH following a dural puncture ranges from $4 \mathrm{~h}$ to 29 weeks ${ }^{2,4}$.

The time that is taken from CSF loss to progress to headache and develop hematoma range from 2 hours to 44 days ${ }^{5}$. Most often when physicians come upon a case of headache following spinal anesthesia, PDPH is assumed to be the cause ${ }^{3,7}$. Headache due to PDPH worsens or develops within 15 minutes after sitting or standing up, and it improves within a similar period after laying down. It appears within 5 days after the puncture and resolves spontaneously within 1 week or up to 48 hours after epidural blood patch ${ }^{8,9}$. This distinguishing feature helps us to exclude other causes of postpartum headache. However, in rare situations, the headache may last for months or even years ${ }^{10}$. ISH should be suspected in a patient when PDPH changes its characteristics to non-postural headache with possible accompanying features like focal central nervous system (CNS) signs, impairment in consciousness level, paresis, ptosis, vomiting, blurring of vision, drowsiness, disorientation, prolonged unresolved headache ${ }^{1,6}$. Unlike these typical features, ISH may present only as a headache and may be unrelated to $\mathrm{PDPH}^{8}$.

Postpartum headaches are quite common $(39 \%)^{4}$, majority of them are primary headaches such as (migraine, tension-type, and cluster headache) and are therefore considered first in the differential diagnosis ${ }^{4}$. Secondary postpartum headache can be fatal and includes PDPH, eclampsia/pre-eclampsia, cerebral venous 
thrombosis, reversible cerebral vasoconstriction syndrome, pituitary mass/hemorrhage ${ }^{4}$. It is common that these headaches coexist and simulate each other in the puerperium causing difficulty in differentiation ${ }^{8}$.

Predisposing factors for ISH following spinal anesthesia are pregnancy, use of large-sized needles, multiple dural punctures dehydration, use of anticoagulants, cerebral vascular abnormalities, and brain atrophy ${ }^{1,2,9}$. Increased susceptibility during pregnancy may be due to differences in elasticity of the dura, hemostatic imbalance, and possibly gender-based differences in cranial morphology ${ }^{1}$. Due to venous dilatation in pregnancy intracranial vessels are prone to tear and bleed. Moreover, postpartum diuresis, peripartum dehydration which could decrease the amount of CSF, sudden reduction of intra-abdominal pressure, vena caval pressure at delivery, hormonally-induced ligamentous changes ${ }^{3}$, Valsalva maneuver at labor ${ }^{3,9}$, thrombocytopenia ${ }^{3,9}$ increases the susceptibility to develop cerebral SDH.

Diagnosis of ISH is usually made by a CT scan of the head. However, cranial Magnetic resonance imaging is more sensitive and specific for iso-dense CSDH ${ }^{7}$. Surgery is indicated if the thickness of hematoma is more than $10 \mathrm{~mm}$, midline shift is greater than $5 \mathrm{~mm}$, or there is neurologic deterioration ${ }^{4}$. In absence of the above features, conservative management is recommended which requires close neurological and radiological follow ups ${ }^{1,2}$. In addition, it is established that ISH caused by dural punctures resulting in long-standing CSF leakage can also be treated with epidural dural patching ${ }^{4}$.

The incidence of ISH following spinal anesthesia and development of related complications is preventable to some extent, vigilance regarding procedure-related factors, prophylactic monitoring of susceptible patients, and regular follow-up after discharge help in avoiding potential morbidity and mortality ${ }^{6}$.

In the reported case the patient developed headache 13 days after LSCS. Her headache did not have an association with PDPH and other neurologic signs. As her symptoms were vague, there was a possibility of misdiagnosis. Perhaps, the development of CSDH in her case was chiefly due to the lumbar puncture during spinal anesthesia. Moreover, her post-pregnancy status may have added up as a predisposing factor for the progression of the CSDH.

\section{CONCLUSIONS:}

ISH is a rare but serious complication following spinal anesthesia and may mimic PDPH. Cases presenting solely with headaches during the postpartum period are often assumed to be benign, and conditions such as ISH may be masked initially resulting in a delay in diagnosis and intervention. Therefore, with proper neurological examination and regular follow up the possibility of an ISH must be kept in mind when evaluating these cases.

\section{ABBREVIATION:}

CNS: Central nervous system

CSDH: Chronic subdural hematoma

CSF: Cerebrospinal fluid

CT: Computed tomography

ISH: Intracranial subdural hematoma

PDPH: Post-dural puncture headache

LSCS: Lower segment cesarean section

SDH: Subdural hematoma

\section{AUTHOR CONTRIBUTION:}

Suyasha Rajbhandari: Original draft, Conceptualization, review and editing

Pritam Gurung: Review, editing and supervision 
Gopi Nepal: Established the diagnosis and treated the patient.

Samir Acharya: Established the diagnosis and treated the patient.

Basant Pant: Supervision

\title{
ACKNOWLEDGEMENTS:
}

The authors express their gratitude to Dr. Saujanya Rajbhandari for proof reading of the manuscript.

\section{CONFLICT OF INTEREST}

None of the authors have potential conflicts of interest to be disclosed.

\section{ETHICS APPROVAL AND CONSENT TO PARTICIPATE}

Ethical approval of case report is not needed in accordance to the local ethical guideline. Written informed consent was obtained from the patient to include the clinical details.

\section{KEY CLINICAL MESSEGE}

Headache is common presentation during postpartum period. Anesthesiologist, obstetrician, and neurosurgeon should be aware about the possibility of subdural hematoma in patient complaining of headache following spinal anesthesia.

\begin{abstract}
Intracranial subdural hematoma following spinal anesthesia is an infrequent occurrence and has variable presentation. Due to rarity, it may often be misdiagnosed as post-dural puncture headache. In this report, we describe a case of a 25-year-old woman who presented with a headache following lower segment cesarean section after spinal anesthesia.
\end{abstract}

Key words

Post-partum, chronic, subdural

\section{References:}

1. Moradi M, Shami S, Farhadifar F, Nesseri K. Cerebral subdural hematoma following spinal anesthesia: Report of two cases. Case Rep Med . 2012;2012. doi:10.1155/2012/352028

2. Lin S-L, Yeh C-C, Wu Z-F, Pan R-Y, Tseng W-C. Intracranial Subdural Hematoma Following Spinal Anesthesia: A Case Report and Literature Review. J Med Sci / Publ by Wolters Kluwer-Medknow J Med Sci . 2020;41(2):92-95. doi:10.4103/jmedsci.jmedsci_104_20

3. Saridogan E. Postpartum subdural hematoma following neuraxial anesthesia: A case report and review of literature. Med Sci . 2019;8(4):1028-1060. doi:10.5455/medscience.2019.08.9055

4. Szeto V, Kosirog J, Eilbert W. Intracranial subdural hematoma after epidural anesthesia: a case report and review of the literature. doi:10.1186/s12245-018-0199-2

5. Brasileira R. INTRACRANIAL SUBDURAL HEMATOMA: A RARE COMPLICATION FOLLOWING SPINAL ANESTHESIA: CASE REPORT . Vol 89.

6. Schweiger V, Zanconato G, Lonati G, Baggio S, Gottin L, Polati E. Intracranial Subdural Hematoma after Spinal Anesthesia for Cesarean Section. Case Rep Obstet Gynecol . 2013;2013:1-3. doi:10.1155/2013/253408

7. Özdem $<\mathrm{r}$ N, Ari MK, Gelal MF, Bez<rc $<\mathrm{O} / \mathrm{lu} \mathrm{H}$, Ataturk İ.Intracranial Chronic Subdural Haematoma as a Complication of Epidural Anesthesia Epidural Anestezinin Komplikasyonu Olarak Geliflen <ntrakranial Kronik Subdural Hematom. Vol 19.; 2009. 
8. Auxiliadora Amorim J, Souza Canuto dos Anjos Remígio D, Damázio Filho O, Aureliano Guerra de Barros M, Nicole Carvalho V, Moraes Valença M.CLINICAL INFORMATION Intracranial Subdural Hematoma Post-Spinal Anesthesia: Report of Two Cases and Review of 33 Cases in the Literature. Vol 60.; 2010.

9. Eldemrdash A, Elsharkawy M, Shams G, Ismail K. Chronic Subdural Hematoma Complicated Spinal Anesthesia: Report and Pilot Study of Five Surgically Treated Cases. doi:10.4172/2155-6148.1000795

10. Kwak KH. Postdural puncture headache. Korean J Anesthesiol . 2017;70(2):136-143. doi:10.4097/kjae.2017.70.2.136

\section{Figure legends:}

Figure 1: Computed tomography scan of the head showing chronic subdural hematoma in right frontoparieto-temporal convexity.

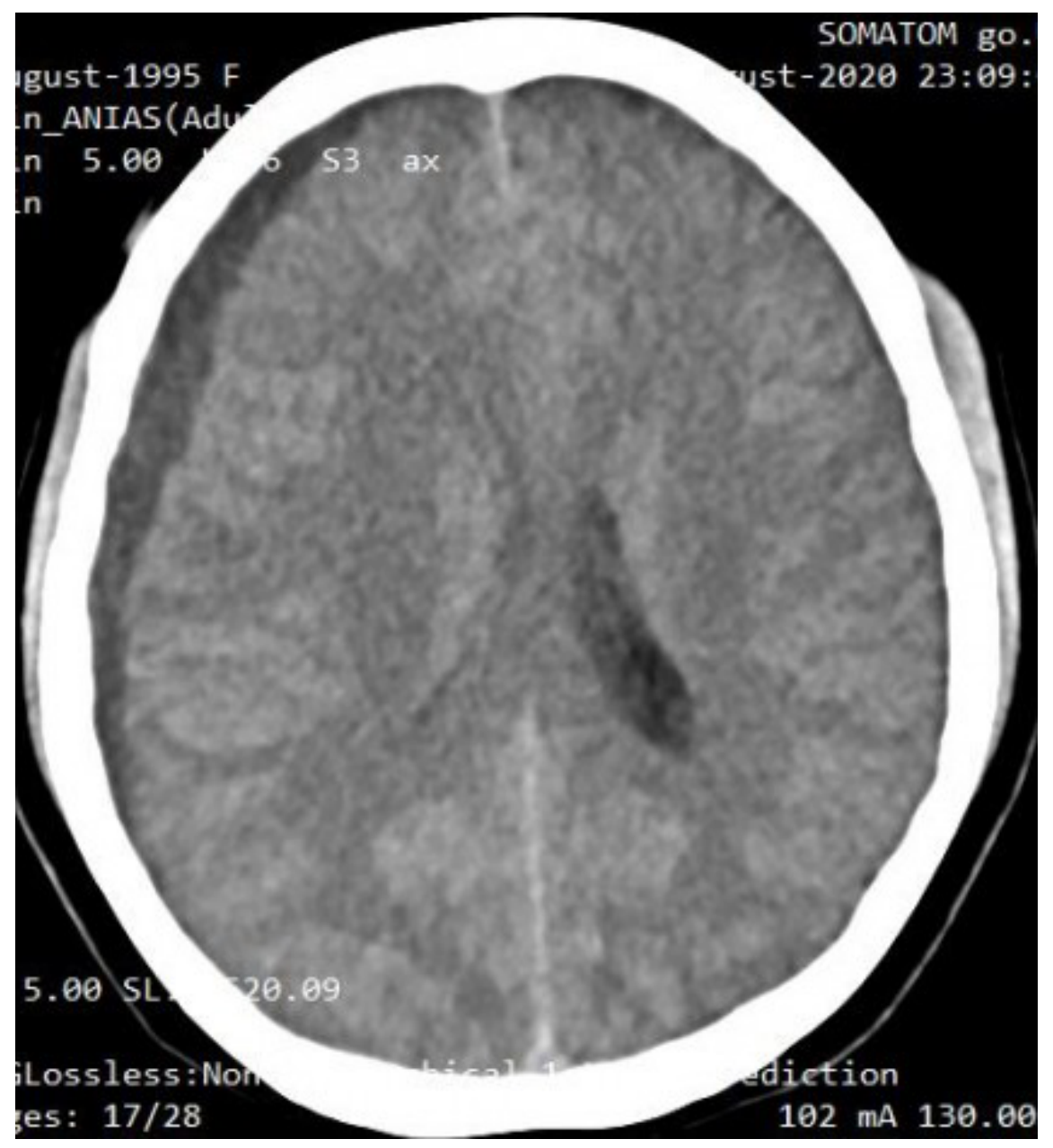

